\title{
Progressive Changes in Glutamate Concentration in Early Stages of Schizophrenia: A Longitudinal 7-Tesla MRS Study
}

\author{
Peter Jeon $^{1,2}$, Roberto Limongi ${ }^{3, \oplus}$, Sabrina D. Ford ${ }^{4}$, Michael Mackinley ${ }^{5}$, Kara Dempster ${ }^{6}$, Jean Théberge ${ }^{1,2,7,8}$, and \\ Lena Palaniyappan*,1,3,4, \\ ${ }^{1}$ Department of Medical Biophysics, Western University, London, Ontario, Canada; ${ }^{2}$ Lawson Health Research Institute, Imaging \\ Division, London, Ontario, Canada; ${ }^{3}$ Robarts Research Institute, Western University, London, Ontario, Canada; ${ }^{4}$ Department of \\ Psychiatry, Western University, London, Ontario, Canada; ${ }^{5}$ Department of Neuroscience, Western University, London, Ontario, \\ Canada; ${ }^{6}$ Department of Psychiatry, Dalhousie University, Halifax, Nova Scotia, Canada; ${ }^{7}$ St. Joseph's Health Care, Diagnostic \\ Imaging, London, Ontario, Canada; ${ }^{8}$ Department of Medical Imaging, Western University, London, Ontario, Canada \\ *To whom correspondence should be addressed; Robarts Research Institute, 1151 Richmond Street N., Room 3208, UWO, London, \\ Ontario, Canada, N6A 5B7; tel: 519-931-5777 (ext. 24398), fax: +1 519 685-8, e-mail: lpalaniy@uwo.ca
}

Progressive reduction in glutamatergic transmission has been proposed as an important component of the illness trajectory of schizophrenia. Despite its popularity, to date, this notion has not been convincingly tested in patients in early stages of schizophrenia. In a longitudinal $7 \mathrm{~T}$ magnetic resonance spectroscopy (1H-MRS), we quantified glutamate at the dorsal anterior cingulate cortex in 21 participants with a median lifetime antipsychotic exposure of less than 3 days and followed them up after 6 months of treatment. Ten healthy controls were also scanned at 2 time points. While patients had significantly lower overall glutamate levels than healthy controls $(F(1,27)=5.23$, $\boldsymbol{P}=.03$ ), we did not observe a progressive change of glutamate concentration in patients $(F(1,18)=0.47, P=.50)$, and the group by time interaction was not significant $(F(1,27)=0.86, P=.36)$. On average, patients with early psychosis receiving treatment showed a $0.02 \mathrm{mM} / \mathrm{y}$ increase, while healthy controls showed a $0.06 \mathrm{mM} / \mathrm{y}$ reduction of MRS glutamate levels. Bayesian analysis of our observations does not support early, post-onset glutamate loss in schizophrenia. Interestingly, it provides evidence in favor of a lack of progressive glutamate change in our schizophrenia sample - indicating that the glutamate level at the onset of illness was the best predictor of the levels 6 months after treatment. A more nuanced view of glutamatergic physiology, linked to early cortical maturation, may be required to understand glutamate-mediated dynamics in schizophrenia.

Key words: first-episode schizophrenia/magnetic resonance spectroscopy/Bayesian

\section{Introduction}

Glutamatergic disruption is implicated in the wide range of symptoms observed in schizophrenia. ${ }^{1-3}$ Specifically, disinhibition of the excitatory glutamatergic outputs of the prefrontal cortex is thought to disrupt dopaminergic signaling in the striatum ${ }^{4,5}$ resulting in acute psychotic symptoms. However, sustained disinhibition of prefrontal glutamatergic neurons might lead to excitotoxic damage with subsequent reduction in glutamate, with greater reductions occurring in patients with more severe forms of schizophrenia. ${ }^{6}$ Magnetic resonance spectroscopy (MRS) studies in schizophrenia report higher glutamate levels in younger patients at early stages while lower glutamate levels in older patients at later stages of illness, when compared to healthy controls (HC). ${ }^{7}$

A progressive pathology defined by gray matter changes, ${ }^{8-10}$ ventricular enlargement, ${ }^{11-15}$ and networklevel dysconnectivity ${ }^{16,17}$ is thought to be the basis of the longitudinal trajectory of schizophrenia. It is posited that glutamatergic dendritic spine reduction triggered by early excitotoxic processes lies at the center of such morphological changes. ${ }^{18,19}$ The progressive pathology of schizophrenia is likely limited to certain hubs of the brain, ${ }^{16}$ with the anterior cingulate cortex (ACC) being a prominent region where both structural, functional, ${ }^{20}$ and neurochemical deficits ${ }^{21-24}$ have been consistently demonstrated in schizophrenia. Nevertheless, longitudinal MRS studies investigating progressive glutamate changes in the ACC are limited.

Using $1.5 \mathrm{~T}$ in patients at various stages of schizophrenia, Choe et $\mathrm{al}^{25}$ reported a notable

(C) The Author(s) 2021. Published by Oxford University Press on behalf of the University of Maryland's school of medicine, Maryland Psychiatric Research Center.

This is an Open Access article distributed under the terms of the Creative Commons Attribution-NonCommercial License (http://creativecommons.org/ licenses/by-nc/4.0/), which permits non-commercial re-use, distribution, and reproduction in any medium, provided the original work is properly cited. For commercial re-use, please contact journals.permissions@oup.com 
reduction in prefrontal Glx (glutamate + glutamine) signal 1-6 months after treatment. Théberge et $\mathrm{al}^{10}$ and Bustillo et $\mathrm{a}^{26}$ demonstrated static glutamate levels in the ACC using 4T. Merritt et $\mathrm{al}^{27}$ also failed to see a progressive reduction in Glx using 3T in schizophrenia. When using 7T MRS, with superior specificity for glutamate quantification, ${ }^{28}$ a cross-sectional association of decreasing ACC glutamate with increasing age was observed in schizophrenia ${ }^{29}$ at an accelerated rate compared to healthy young adults. ${ }^{30}$ Nevertheless, to date, longitudinal 7T MRS studies have not been reported in schizophrenia.

In this study, we tested if (1) glutamate-mediated deficit indexed by ACC MRS measure of glutamate is present in early stages of psychosis, (2) whether this deficit progressively worsens in the first 6 months of treatment, and (3) if patients show an exaggerated longitudinal decline compared to HC. To our knowledge, this is the first longitudinal report of 7T MRS in schizophrenia.

\section{Methods}

\section{Participants}

We recruited 21 first-episode schizophrenia (FES) volunteers with inclusion criteria of lifetime antipsychotic exposure being less than 14 days along with 10 healthy control volunteers, group-matched for age, gender, and parental socio-economic status. Patient volunteers were recruited from the referrals received by the PEPP (Prevention and Early Intervention for Psychosis Program) at London Health Sciences Center. All patients had established consensus diagnosis after 6 months of FES by 3 psychiatrists (L.P., K.D., and primary treatment provider at PEPP) based on the DSM-5 criteria. ${ }^{31}$ Participants whose 6-month diagnoses were bipolar or major depressive disorder with psychoses as well as suspected drug-induced psychoses were excluded from the study. Healthy control volunteers had no personal history of mental illness and no family history of psychotic disorder. All participants were screened to exclude significant head injury, major medical illness, or MRI contraindications and provided written, informed consent according to the guidelines of the Human Research Ethics Board for Health Sciences at Western University, London, Ontario.

\section{MRS Acquisition and Analysis}

MRS measurements were acquired using a Siemens MAGNETOM 7T head-only MRI scanner (Siemens, Erlangen, Germany) and a site-built head coil (8-channel transmit, 32-channel receive) at the Centre for Functional and Metabolic Mapping of Western University (London, Ontario). A 2-dimensional sagittal anatomical image (37 slices, $\mathrm{TR}=8000 \mathrm{~ms}, \mathrm{TE}=70 \mathrm{~ms}$, flip-angle $(\alpha)=120^{\circ}$, thickness $=3.5 \mathrm{~mm}$, field of view $=240 \times 191 \mathrm{~mm}$ ) was used as reference to prescribe a $2.0 \times 2.0 \times 2.0 \mathrm{~cm}$ $\left(8 \mathrm{~cm}^{3}\right){ }^{1} \mathrm{H}-\mathrm{MRS}$ voxel on the bilateral dorsal ACC (figure 1). Voxel positioning was set by having the posterior end of the voxel coinciding with the precentral gyrus and the caudal face of the voxel coinciding with the most caudal positioning that was not part of the corpus callosum. Voxel angle was set to be tangential to the corpus callosum. A semi-LASER ${ }^{1} \mathrm{H}-\mathrm{MRS}$ sequence $(\mathrm{TR}=7500 \mathrm{~ms}, \mathrm{TE}=100 \mathrm{~ms}$, bandwidth $=6000 \mathrm{~Hz}$, $N=2048$ ) was used to acquire 32 channel-combined, VAPOR 32 water-suppressed spectra as well as a waterunsuppressed spectrum to be used for spectral editing and quantification. During scan, participants were asked to rest by fixing their gaze on a white cross on a $50 \%$ gray background.

Using the tools outlined in Near et al, ${ }^{33}$ the 32 spectra were phase and frequency corrected before being averaged into a single spectrum to be used for all subsequent analyses. QUECC34 and HSVD35 were applied to the spectrum for lineshape deconvolution and removal of residual water signal, respectively. Spectral fitting was done using fitMAN, ${ }^{36}$ a time-domain fitting algorithm that uses a nonlinear, iterative Levenberg-Marquardt minimization algorithm to estimate the chemical shift, amplitude, linewidth and phase of echo time-specific prior knowledge templates. The metabolite fitting template included 17 brain metabolites: alanine, aspartate, choline, creatine, $\gamma$-aminobutyric acid (GABA), glucose, glutamate, glutamine, glutathione, glycine, lactate, myoinositol, $\mathrm{N}$-acetyl aspartate, $\mathrm{N}$-acetyl aspartyl glutamate, phosphorylethanolamine, scyllo-inositol, and taurine. No significant macromolecule contribution was expected due to the long echo time and hence was omitted from the metabolite template. Metabolite quantification was then performed using Barstool ${ }^{37}$ with corrections made for tissue-specific (gray matter, white matter, CSF) $\mathrm{T}_{1}$ and $T_{2}$ relaxations through partial volume segmentation calculations of voxels mapped onto $T_{1}$-weighted images acquired using a $0.75 \mathrm{~mm}$ isotropic MP2RAGE sequence $\left(\mathrm{TR}=6000 \mathrm{~ms}, \mathrm{TI}_{1}=800 \mathrm{~ms}, \mathrm{TI}_{2}=2700 \mathrm{~ms}\right.$, flip-angle 1 $\left(\alpha_{1}\right)=4^{\circ}$, flip-angle $2\left(\alpha_{2}\right)=5^{\circ}, \mathrm{FOV}=350 \mathrm{~mm} \times 263 \mathrm{~mm}$ $\times 350 \mathrm{~mm}, T_{\text {acq }}=9$ min $38 \mathrm{~s}$, iPAT $_{\mathrm{PE}}=3$ and $6 / 8$ partial $\mathrm{k}$-space). All spectral fit underwent visual quality inspection as well as Cramer-Rao lower bounds (CRLB) assessment for each metabolite and fit parameter.

\section{Clinical Assessments}

Symptom severity was measured using PANSS- $8^{38}$ scale, on the same day as the first scan as well as the follow-up scan. We also quantified the overall social and occupational functioning at the time of first presentation using SOFAS, ${ }^{39}$ also administered on both baseline and follow-up scan days. We assessed Duration of Untreated Psychosis (DUP) based on multiple sources of information provided by the patient, the referring sources, and 

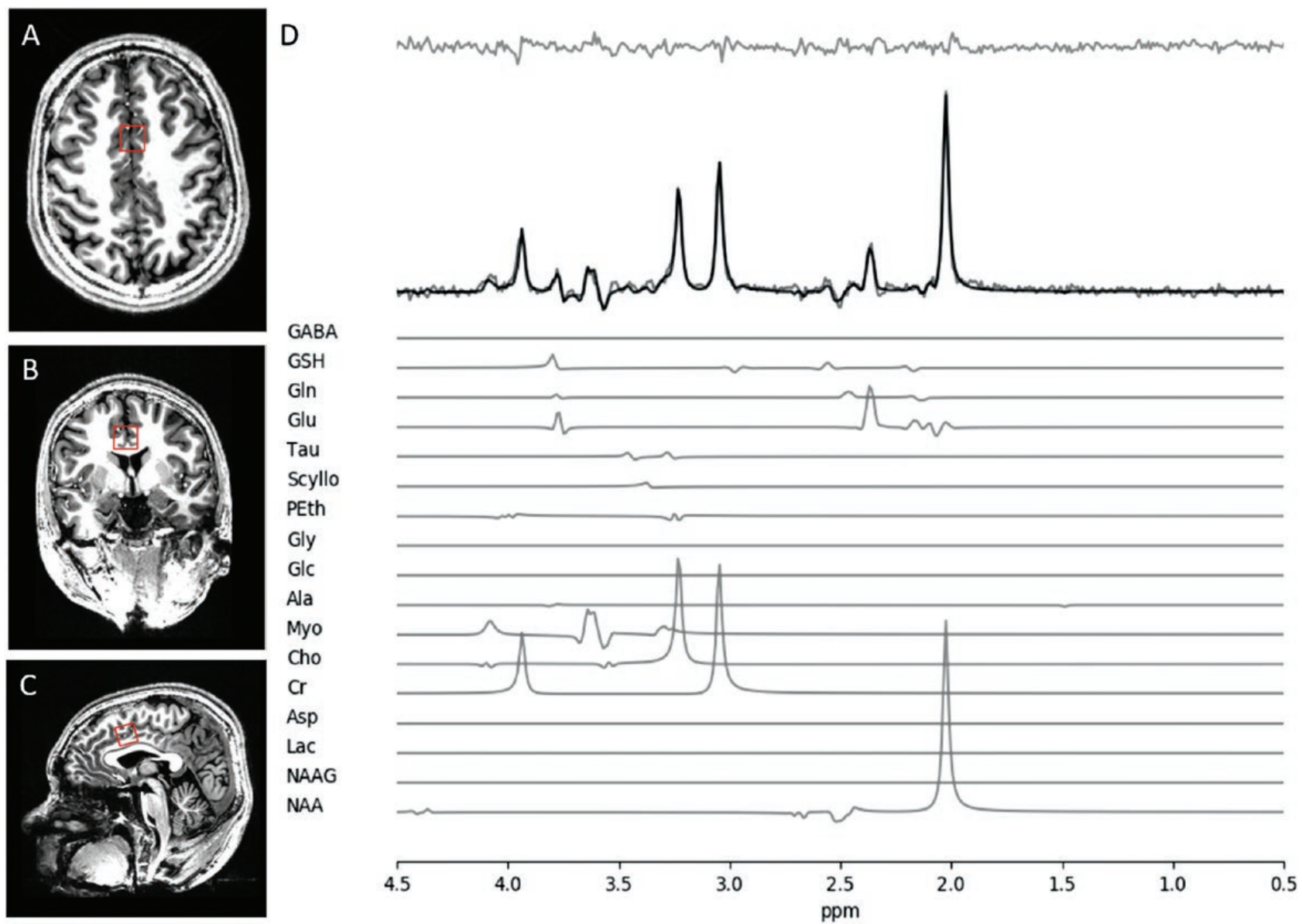

Fig. 1. (A) Axial, (B) coronal, and (C) sagittal views of MRS voxel (red square) in the dorsolateral anterior cingulate cortex (ACC) for glutamate measurement. (D) Sample fitted spectrum of a single participant. Fit spectrum (bolded) is overlaid on the raw spectrum with the residual spectrum displayed above. Individual component spectra of all 17 template-included metabolites are displayed below.

caregivers as well as by reviewing clinical charts. We used the first emergence of positive psychotic symptoms as the starting point for calculating the DUP, in line with prior work in this regard. ${ }^{40}$

To determine cannabis use in the past 6 months, the Cannabis Abuse Screening Test (CAST) was used. ${ }^{41}$ The CAST is a 6-item Likert-scale self-report questionnaire which asks the participant about cannabis use and how it effects their daily activities and relationships. Scores range from 6 to 30, with higher scores indicating more cannabis use. To determine alcohol use in the past 6 months, the Alcohol Use Disorders Identification Test (AUDIT-C) ${ }^{42}$ was used. The AUDIT-C is a 3-item Likert-scale self-report questionnaire that asks the participant about alcohol use frequency and quantity. Scores range from 0 to 12 , with higher scores indicating more alcohol use. Alcohol users and nonusers were classified by AUDIT-C scores of 4 or more and less than 4, respectively. Lastly, nicotine use in the past 6 months was determined by the single item Fagerström Test for Nicotine Dependence and smoking index. ${ }^{43}$ The Fagerström test indicates time to the first cigarette after waking, and the smoking index is calculated by the number of years regularly smoking $\times$ the number of cigarettes per day, divided by 20, to determine packs per year. A lower Fagerström test value indicates more nicotine dependence, and a higher smoking index indicates more nicotine use. The 10-item Drug Abuse Screening Test (DAST-10) ${ }^{44}$ was also employed for substances other than cannabis, alcohol and nicotine, though our cohort did not endorse any such use.

\section{Statistical Analyses}

All frequentist statistical tests were computed using IBM SPSS Statistics version 26. ${ }^{45}$ Group demographic differences were calculated using $t$ tests and chi-square tests for continuous and dichotomous variables, respectively. Repeated measure ANOVA was used to assess group $\times$ time interaction (primary hypothesis), as well as group effect and time effect, with parameter estimates examined to test individual group effects. As age and gender are known modifiers of glutamate levels, they were entered as covariates in the ANOVA model. Lastly, Pearson correlation was used to explore the correlation of annualized, baseline-adjusted glutamate change to DUP, SOFAS, as well as symptom 
severity at first presentation and follow-up, measured using PANSS-8 total score at respective time points in patients. Correlations between defined daily dose (DDD) and annualized, baseline-adjusted glutamate change and follow-up glutamate concentrations were also examined.

To investigate relationships of annualized glutamate with cannabis and nicotine, Pearson correlations were used. Glutamate change was analyzed with total CAST to determine relationships with cannabis, along with smoking index and Fagerström scores to determine nicotine use. To determine alcohol use, a $t$-test was used to compare glutamate change values between alcohol users and nonusers.

We performed a (Bayesian) hierarchical generalization of an analysis of covariance (Bayesian ANCOVA) to evaluate whether there is a between-group difference of the effect of time on the follow-up measurement of glutamate concentration. We decided to use a Bayesian approach as an alternative to traditional frequentist test because it allows us to weigh the evidence in support of our main hypothesis relative to the evidence in support of the null hypothesis. We achieved this via model comparison and Bayes factors (BF). ${ }^{46,47}$

We fit one saturated ANCOVA model and all possible reduced models comprising follow-up glutamate concentration $\left([\mathrm{Glu}]_{\text {follow-up }}\right.$ ) as a dependent variable, Group as factor, and baseline glutamate concentration ([Glu $]_{\text {baseline }}$ ) and Interval (days) as covariates. We compared the evidence supporting this (saturated) model with the evidence supporting the reduced models (including the null model). We relied on the largest $\mathrm{BF}_{10}$ relative to the null model to select the winning model. Note that we included $[\mathrm{Glu}]_{\text {baseline }}$ as a covariate of no interest. Therefore, our main hypothesis was represented by the triple-interaction model $\left(\right.$ Group $\times[\mathrm{Glu}]_{\text {baseline }} \times$ Interval $)$.

In all models, the posterior distributions over parameters were estimated using the "generaltestBF" function in the "R Bayes Factor" package. ${ }^{48}$ In this data set, the small number of subjects in each group might cause the posterior distribution to be strongly influenced by the prior distribution. Therefore, we used informed "wide" priors scaled to the observed data ( $r$-scale for each effect $=0.5$ ). We report the mean and standard deviation for each estimate obtained from the relevant posterior distribution (10 000 samples) along with the 95\% highest density interval (HDI).

\section{Results}

\section{Demographic Data}

Demographic and clinical data of subjects are shown in table 1. Our patient sample had a mean DUP of 29.38 weeks $(\mathrm{SD}=26.65 \mathrm{wk})$ and a mean antipsychotic duration of 2.95 days $(\mathrm{SD}=3.11 \mathrm{~d})$ prior to the first scan session. Patient and healthy control SOFAS scores were significantly different $(t(29)=12.466, P<.001)$. The time in between baseline and follow-up (FUP) scan was 5.93 months $(\mathrm{SD}=1.25)$ for patients and 7.25 months $(\mathrm{SD}=1.90)$ for $\mathrm{HC}$.

CRLB values indicating the quality of glutamate measurement was quantified for both groups. For HC glutamate CRLB were $3.41 \%(\mathrm{SD}=1.27 \%)$ and $3.55 \%(\mathrm{SD}=0.89 \%)$ for baseline and FUP, respectively. For FES glutamate

Table 1. Demographic and Clinical Characteristics

\begin{tabular}{|c|c|c|c|c|}
\hline Characteristic & Patient Group $(N=21)$ & Healthy Controls $(N=10)$ & $t / \chi^{2}$ & $P$ \\
\hline Gender (male/female) & $16 / 5$ & $5 / 5$ & 2.13 & 0.145 \\
\hline Marital status (Mar/S) & $1 / 20$ & $1 / 9$ & 0.31 & 0.58 \\
\hline Inpatient at baseline $(\mathrm{Y} / \mathrm{N})$ & $11 / 10$ & & & \\
\hline Family $\mathrm{Hx}(\mathrm{Y} / \mathrm{N} / \mathrm{DN})$ & $10 / 6 / 5$ & & & \\
\hline AP exposure at baseline (M/SD; days) & $2.95 / 3.11$ & & & \\
\hline Total DDD-days at baseline scan (M/SD) & $2.25 / 4.74$ & & & \\
\hline Total DDD-days at FUP scan (M/SD) & $145.68 / 97.56$ & & & \\
\hline DUP (M/SD/median; wk) & $29.38 / 26.65 / 18$ & & & \\
\hline Ethnicity (Black/White/Other) & $2 / 18 / 1$ & $0 / 5 / 5$ & 4.51 & $0.034^{\mathrm{a}}$ \\
\hline Age (M/SD) & $22.33 / 5.29$ & $21.60 / 3.37$ & -0.47 & 0.645 \\
\hline SOFAS at baseline scan (M/SD) & $42.33 / 12.84$ & $83.70 / 5.62$ & 12.47 & 0.000 \\
\hline SOFAS at FUP scan (M/SD) & $61.25 / 9.85$ & $85.10 / 3.21$ & 9.83 & 0.000 \\
\hline PANSS-8 total at baseline scan (M/SD) & $24.67 / 5.30$ & & & \\
\hline PANSS-8 total at FUP scan (M/SD) & $14.35 / 4.77$ & & & \\
\hline Smoker (yes/no) & $6 / 15$ & $0 / 10$ & 3.54 & 0.060 \\
\hline Cannabis user (yes/no) & $13 / 8$ & $0 / 10$ & 10.66 & 0.001 \\
\hline Time between scans (M/SD; mo) & $5.93 / 1.25$ & $7.67 / 1.90$ & 2.63 & 0.021 \\
\hline
\end{tabular}

Note: Mar, married; S, single; Y, yes; N, no; Hx, history; DN, don't know; AP, antipsychotic; M, mean; DDD, defined daily dose; FUP, follow-up; DUP, duration untreated psychosis. $P$ values for differences between groups were calculated using chi-square analyses for categorical variables and independent $t$ tests for continuous variables.

${ }^{a}$ White vs non-White comparison.

Page 4 of 9 
quantification, CRLB values were $3.52 \%(\mathrm{SD}=1.20 \%)$ and $3.96 \%(\mathrm{SD}=1.12 \%)$ for baseline and FUP, respectively. Thus the 2 groups had acceptable qualitative metrics for glutamate estimation at both time points. A sample of fitted spectrum is presented in figure 1 . We present the concentration and CRLB of metabolites other than glutamate in the Supplementary Material.

\section{Longitudinal Glutamate}

Repeated measures ANOVA revealed a group effect $(F(1,27)=5.23, P=.03$, Cohen's $d=0.90)$ between FES $(M=6.43 \mathrm{mM}, \mathrm{SD}=0.84 \mathrm{mM})$ and $\mathrm{HC}(M=7.20 \mathrm{mM}$, $\mathrm{SD}=0.86 \mathrm{mM})$ but no effect on time $(F(1,27)=1.21$, $P=.28)$ or group $\times$ time interaction $(F(1,27)=0.86$, $P=.36)$. Parameter estimates revealed that at baseline, FES had lower glutamate than $\mathrm{HC}(t(29)=2.83, P=.009$, Cohen's $d=1.11)$, but this difference was not present at follow-up $(t(29)=1.20, P=.24$, Cohen's $d=0.41)$. A simple contrast of time in each group revealed no significant effect in both the healthy control group $(F(1,7)=0.25$, $P=.63$, Cohen's $d=0.41)$ and in patients $(F(1,18)=0.47$, $P=.50$, Cohen's $d=.003$ ) (figure 2).

Annualized glutamate concentration values were not significantly different between the 2 groups $(t(29)=-0.813, P=.423)$ and indicated a $0.02 \mathrm{mM} / \mathrm{y}$ $(\mathrm{SD}=0.33 \mathrm{mM})$ increase in patients and a $0.06 \mathrm{mM} / \mathrm{y}$ $(\mathrm{SD}=0.19)$ reduction in healthy controls, with the difference amounting to a small to moderate sized effect (Cohen's $d=0.26$ ). Lastly, the time interval between scans in months was not related to glutamate concentration differences (FUP - baseline) in either group (HC: $r=-.23, P=.52$; FES: $r=-.41, P=.06$ ).

\section{Glutamate vs Clinical Measures}

There was no significant correlation between annualized glutamate concentration changes and DUP $(r=-.07$, $P=.77)$, SOFAS at baseline $(r=-.09, P=.70)$, PANSS-8

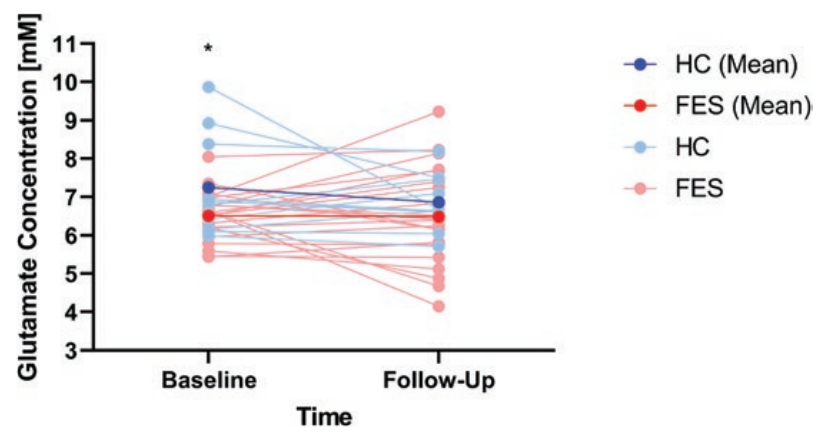

Fig. 2. Means of glutamate concentration $[\mathrm{mM}]$ for healthy controls (HC, blue) and patients (FES, red) at baseline and follow-up scan sessions. Subject-level glutamate changes are shown in light blue for healthy controls and pink for patients. Asterisk denotes significant difference between means (Note: $y$-axis values do not begin at 0 for graphical purposes). total at baseline $(r=.25, P=.28)$, SOFAS at follow-up $(r=.36, P=.12)$, or PANSS-8 total at follow-up $(r=.05$, $P=.84)$. We also did not see any correlation between baseline (unadjusted) glutamate concentration and DUP $(r=.03, P=.91)$, SOFAS $(r=-.38, P=.09)$, or PANSS-8 total $(r=.31, P=.17)$.

Across all participants, there was no significant difference in annualized glutamate change between alcohol users $(n=23)$ and nonusers $(n=6)(t(27)=1.89, P=.07)$. No significant difference was found between alcohol users and nonusers when FES was considered $(t(17)=1.37$, $P=.19)$ separately. No significant difference was found in annualized glutamate change between smokers and non-smokers among the FES patients $(t(20)=-.72$, $P=0.63)$. Across all participants, there was no significant correlation between total CAST scores $(n=28)$ and annualized glutamate change $(r=-.08, P=.75)$. Lastly, there was a significant correlation between DDD at baseline $(n=21)$ and annualized glutamate change $(r=-.44$, $P=.04)$. There was no significant correlation between DDD at baseline and baseline glutamate concentration $(r=.11, P=.64)$, between DDD at follow-up $(n=21)$ and follow-up glutamate concentration $(r=-.16, P=.50)$, as well as between DDD at follow-up and annualized glutamate change $(r=-.16, P=.48)$.

\section{Bayesian Statistical Analysis}

After controlling for baseline values, the $[\mathrm{Glu}]_{\text {follow-up }}$ in the FEP group was the same as in the HC group. The Bayesian ANCOVA model revealed that the triple interaction $\left(\right.$ Group $\times[\mathrm{Glu}]_{\text {baseline }} \times$ Interval) did not perform better than the null model (table 2). This means that, in

Table 2. Bayesian Model Comparison

\begin{tabular}{ll}
\hline Model & $B_{10}$ \\
\hline N & 1.000 \\
$G$ & 0.465 \\
B & $\mathbf{4 . 0 8 0}$ \\
$G+B$ & 1.547 \\
$G+B+G \times B$ & 1.732 \\
I & 0.351 \\
$G+I$ & 0.201 \\
B + I & 3.126 \\
$G+B+I$ & 1.451 \\
$G+B+G \times B+I$ & 1.828 \\
$G+I+G \times I$ & 0.155 \\
$G+B+I+G \times I$ & 0.905 \\
$G+B+G \times B+I+G \times I$ & 1.929 \\
B + I + B $\times$ I & 3.164 \\
$G+B+I+B \times I$ & 1.448 \\
$G+B+G \times B+I+B \times I$ & 0.980 \\
$G+B+I+G \times I+B \times I$ & 2.855 \\
$G+B+G \times B+I+G \times I+B \times I$ & 1.814 \\
$G+B+G \times B+I+G \times I+B \times I+G \times B \times I$ & 0.960 \\
\hline
\end{tabular}

Note: $\mathrm{BF}_{10}$ is computed relative to null model. $N$ null, $B$ baseline, $I$ interval, $G$ group. 
practice, the longitudinal change in glutamate concentration of both groups are alike.

Table 2 also shows that a model including only the $[\mathrm{Glu}]_{\text {baseline, }}$, model including both $[\mathrm{Glu}]_{\text {baseline }}$ and Interval, and a model including its interaction outperformed the null model $\left(\mathrm{BF}_{10}>3\right)$. However, the more complex models underperformed the " $[\mathrm{Glu}]_{\text {baseline }}$ " model (the best model). This suggests that, with moderate evidence (10 > $\left.\mathrm{BF}_{10}>3\right),{ }^{47}[\mathrm{Glu}]_{\text {baseline }}$ is the best predictor of $[\mathrm{Glu}]_{\text {follow-up }}$ regardless of both follow-up measurement time and Group. Table 3 shows the relevant parameter estimates along with the $95 \%$ interval of most credible values.

\section{Discussion}

We report longitudinal 7T MRS glutamate measurements in FES patients who were medication-naïve at baseline. Although baseline glutamate concentrations were lower in FES compared to HC, follow-up measurements revealed no difference in glutamate concentration between the 2 groups. Our current work supports the lower glutamate concentration observed in FES compared to HC at baseline as reported in current literature. ${ }^{49}$ Annualized glutamate concentration changes also showed no difference between FES and HC. Bayesian statistical approach also provided evidence in favor of a lack of progressive glutamate change in our FES sample, indicating that the glutamate-mediated level at the onset of illness was the best predictor of the levels 6 months after treatment. Taken together, our results indicate that early in the illness, patients with schizophrenia already show abnormalities in ACC glutamate (baseline), which may implicate low glutamatergic synapse density, reduced neuroexcitatory transmission, or imbalance of glutamatergic activity, ${ }^{49}$ and do not show evidence for a progressive deterioration in glutamate-mediated status within the first 6 months.
We observed a reduction in glutamate in the untreated, acute stage of schizophrenia. While Meritt and colleagues showed no elevation of medial frontal glutamate+glutamine (Glx signal) in schizophrenia, ${ }^{2}$ Sydnor and colleagues reported a reduction in glutamate signal from 7T MRS49 studies in patients compared to controls. While these findings are somewhat counterintuitive to the prevailing notion of glutamatergic excitotoxicity in acute psychosis, there are at least 3 possible explanations. Firstly, it is possible that glutamatergic excess predates acute psychosis, and is short-lived as compensatory metabolic pathways reset the imbalance by the time the first clinical presentation occurs. This is supported by meta-analytic observations that medial prefrontal Glx is higher in high-risk subjects. ${ }^{2,50}$ Secondly, it is possible that the excitotoxity is spatially constrained to regions other than the medial prefrontal cortex (eg, hippocampus or thalamus) though MRS studies have so far failed to show a consistent elevation of glutamate in these regions across various stages of schizophrenia. . $^{2,50-52}$ Finally, it is possible that the resting-state level of MRS glutamate or glutamine (as shown in Supplementary Materials) do not capture the suspected excitotoxic process; dynamic measures of glutamate response for task-related demands (as in fMRS) may be required for a more complete picture.

Prior longitudinal studies at lower field strengths have been equivocal on the issue of progressive glutamatergic reduction in schizophrenia. ${ }^{7}$ Synthesizing this longitudinal literature, Egerton et $\mathrm{al}^{53}$ noted an almost even split between studies that reported significant glutamate decrease in at least one brain region and studies reported no glutamate reduction, with at least 3 further studies showing no longitudinal glutamate reduction in the ACC after 6 weeks, ${ }^{27,54,55} 4$ months, ${ }^{54}$ and 9 months. ${ }^{27}$ Our findings are more aligned with these

Table 3. Model Averaged Posterior Summary

$95 \%$ HDI

\begin{tabular}{lrrr} 
Parameter & Mean & SD & Lower \\
\hline Intercept & $\mathbf{6 . 6 1 7}$ & Upper \\
FEP & -0.074 & $\mathbf{0 . 2 0 5}$ & $\mathbf{6 . 2 0 9}$ \\
HC & 0.074 & 0.178 & -0.434 \\
Baseline & $\mathbf{0 . 5 1 1}$ & 0.178 & -0.270 \\
Interval & -0.004 & $\mathbf{0 . 2 4 7}$ & 0.270 \\
FEP $\times[G l u]_{\text {baseline }}$ & 0.149 & 0.004 & -0.012 \\
HC $\times[G l u]_{\text {baseline }}$ & -0.149 & 0.225 & -0.288 \\
FEP $\times$ Interval & -0.006 & 0.225 & -0.595 \\
HC $\times$ Interval & 0.006 & 0.004 & -0.014 \\
{$[\mathrm{Glu}]_{\text {baseline }} \times$ Interval } & -0.005 & 0.004 & -0.001 \\
FEP $\times$ Interval $\times[G l u]_{\text {baseline }}$ & -0.002 & 0.006 & -0.016 \\
HC $\times$ Interval $\times[G l u]_{\text {baseline }}$ & 0.002 & 0.005 & 0.003 \\
$\boldsymbol{\sigma}^{2}$ & $\mathbf{0 . 9 6 4}$ & 0.005 & 0.001 \\
\hline
\end{tabular}

Note: Only the Intercept, $[\mathrm{Glu}]_{\text {baseline }}$ and sig2 parameters (bolded) were granted with $\mathrm{PP}>0.95 . \sigma^{2}$ posterior variance of the "deflection parameters", $P P$ posterior probability.

Page 6 of 9 
recent studies as well as the lack of progressive ACC glutamate change reported by Bustillo et $\mathrm{al}^{26}$ (at 1,6 , 12 months) and Théberge et $\mathrm{al}^{10}$ (10 months) at 4T. In contrast, Egerton et $\mathrm{al}^{56}$ observed a reduction in glutamate to creatine ratios at $3 \mathrm{~T}$ in the ACC after 4 weeks of antipsychotic treatment, though 2 of the 3 sites in this multi-site study did not show the same effect. Kubota and colleagues ${ }^{57}$ recently synthesized the longitudinal MRS studies (1.5, 3, and 4T) in schizophrenia and reported no significant glutamate (or glutamine) reduction over a median of 3.8 months, but the composite Glx signal decreased over time when the data from all frontal regions (white and gray matter, medial and lateral) were combined. Most of the ACC-focused studies in Kubota and colleagues' meta-analysis comprised of cohorts with chronic schizophrenia. It is possible that patients with chronic illness are more likely to have glutamatergic deficits in the ACC compared to an unbiased sample of first-episode patients followed up over time, as in our sample. In summary, our current study supports the extant literature on the lack of progressive glutamate changes in the ACC during the early course of treatment in schizophrenia.

Our study has a number of strengths, including the use of 7T scanner, and recruiting highly symptomatic, mostly drug-naïve individuals. Several limitations should also be considered when interpreting the results. We chose a single voxel (dorsal ACC) for the current 6-month follow-up study. We cannot exclude the possibility of progressive glutamate changes in different brain regions (as shown by Théberge et $\mathrm{al}^{10}$ and Goto et $\mathrm{al}^{58}$ in thalamus and basal ganglia) or in ACC over a longer time scale with multiple time points. MRS glutamine signal is considered to reflect the synaptic pool of glutamate due to the glial glutamine synthesis in response to synaptic release of glutamate. Our acquisition was not optimized for quantifying glutamine; we had high CRLB values $(23.70 \pm 15.93 \%$ and $26.83 \pm$ $12.06 \%$ for baseline and follow-up, respectively) for glutamine quantification. Nevertheless, we did not see progressive glutamine changes in patients (Supplementary Materials), in keeping with our glutamate results.

Although we had sufficient power to detect if paired differences were present in the patient group, any interaction is likely underpowered. We observed a small glutamate decrease in healthy control $(0.06 \mathrm{mM} / \mathrm{y})$, comparable to the effect size observed in Birur et a ${ }^{54}$ with 16 patients and 14 HC. Bayesian statistical approaches are based on an expected prior distribution and not influenced by the central limit of sample size placed on conventional statistics. Bayesian approach also confirmed the evidence in favor of a lack of progressive glutamate-mediated changes in schizophrenia. We acknowledge that the exposure to treatment over 6 months period might have annulled a likely progressive glutamatergic reduction in patients. For obvious ethical reasons, we lacked a patient group that remained untreated for 6 months to parse the effects of treatment from illness stage. Finally, we noted a trend towards higher glutamate reduction with longer interval between the scans in patients, but not in the healthy control group. The possibility of a delayed deficit effect (ie, exaggerated glutamate reduction over a longer period of time in patients compared to controls) requires further investigation in long-term follow-up studies.

In summary, this longitudinal 7T MRS study of ACC found a stable glutamatergic deficit that does not progressively worsen in the early stages of schizophrenia. This supports the possibility that the putative excitotoxic processes predate the first presentation of psychosis, either in the prodromal stages or more distally during early development. Further, this also challenges the notion of a relentlessly progressive glutamate-mediated dysfunction in patients receiving treatment.

\section{Supplementary Material}

Supplementary data are available at Schizophrenia Bulletin Open online.

\section{Funding}

This study was funded by CIHR Foundation Grant (375104/2017) to L.P.; Schulich School of Medicine Clinical Investigator Fellowship to K.D.; AMOSO Opportunities fund to L.P.; BrainSCAN to R.L.; Parkwood Institute Studentship to MM; Canada Graduate Scholarship to K.D. Data acquisition was supported by the Canada First Excellence Research Fund to BrainSCAN, Western University (Imaging Core); Innovation fund for Academic Medical Organization of Southwest Ontario; Bucke Family Fund, The Chrysalis Foundation and The Arcangelo Rea Family Foundation (London, Ontario).

\section{Acknowledgments}

We thank Mr. Trevor Szekeres, Mr. Scott Charlton, Mr. Joseph Gati for their assistance in data acquisition and archiving. We thank Dr. Rob Bartha and Dr. Dickson Wong for consultation provided on MRS analysis. We thank all research team members of the NIMI lab and all the staff members of the PEPP London team for their assistance in patient recruitment and supporting clinical care. We gratefully acknowledge the participants and their family members for their contributions. The MRS data is available upon request for further analysis. Requests for data should be addressed to L.P. "Ipalaniy@ uwo.ca." L.P. reports personal fees from Otsuka Canada, SPMM Course Limited, UK, Canadian Psychiatric Association; book royalties from Oxford University Press; investigator-initiated educational grants from Janssen Canada, Sunovion and Otsuka Canada outside the submitted work. All other authors report no relevant conflicts. 


\section{References}

1. Coyle JT. Glutamate and schizophrenia: beyond the dopamine hypothesis. Cell Mol Neurobiol. 2006;26(4-6):365-384.

2. Merritt K, Egerton A, Kempton MJ, Taylor MJ, McGuire PK. Nature of Glutamate alterations in schizophrenia: a metaanalysis of proton magnetic resonance spectroscopy studies. JAMA Psychiatry. 2016;73(7):665-674.

3. Javitt DC, Zukin SR. Recent advances in the phencyclidine model of schizophrenia. Am $J$ Psychiatry. 1991;148(10):1301-1308.

4. McCutcheon RA, Krystal JH, Howes OD. Dopamine and glutamate in schizophrenia: biology, symptoms and treatment. World Psychiatry. 2020;19(1):15-33.

5. Limongi R, Mackinley M, Dempster K, Khan AR, Gati JS, Palaniyappan L. Frontal - striatal connectivity and positive symptoms of schizophrenia: implications for the mechanistic basis of prefrontal rTMS. Eur Arch Psychiatry Clin Neurosci. 2020;(0123456789). doi:10.1007/ s00406-020-01163-6

6. Kumar J, Liddle EB, Fernandes CC, et al. Glutathione and glutamate in schizophrenia: a 7T MRS study. Mol Psychiatry. 2020;25(4):873-882.

7. Marsman A, Van Den Heuvel MP, Klomp DWJ, Kahn RS, Luijten PR, Hulshoff Pol HE. Glutamate in schizophrenia: a focused review and meta-analysis of 1H-MRS studies. Schizophr Bull. 2013;39(1):120-129. doi:10.1093/schbul/ sbr069

8. Gur RE, Cowell PE, Latshaw A, et al. Reduced dorsal and orbital prefrontal gray matter volumes in schizophrenia. Arch Gen Psychiatry. 2000;57(8):761-768. doi:10.1001/ archpsyc.57.8.761

9. Zipursky RB, Lim KO, Sullivan EV, Brown BW, Pfefferbaum A. Widespread cerebral gray matter volume deficits in schizophrenia. Arch Gen Psychiatry. 1992;49(3):195205. doi:10.1001/archpsyc.1992.01820030027004

10. Théberge J, Williamson KE, Aoyama N, et al. Longitudinal grey-matter and glutamatergic losses in first-episode schizophrenia. Br J Psychiatry. 2007;191:325-334.

11. Weinberger DR, Torrey EF, Neophytides AN, Wyatt RJ. Lateral cerebral ventricular enlargement in chronic schizophrenia. Arch Gen Psychiatry. 1979;36(7):735-739. doi:10.1001/archpsyc.1979.01780070013001

12. Weinberger DR, Bigelow LB, Kleinman JE, Klein ST, Rosenblatt JE, Wyatt RJ. Cerebral ventricular enlargement in chronic schizophrenia. An association with poor response to treatment. Arch Gen Psychiatry. 1980;37(1):11-13.

13. Andreasen NC, Smith MR, Jacoby CG, Dennert JW, Olsen SA. Ventricular enlargement in schizophrenia: definition and prevalence. Am J Psychiatry. 1982;139(3):292-296. doi:10.1176/ajp.139.3.292

14. Andreasen NC, Olsen SA, Dennert JW, Smith MR. Ventricular enlargement in schizophrenia: relationship to positive and negative symptoms. Am J Psychiatry. 1982;139(3):297-302. doi:10.1176/ajp.139.3.297

15. Reveley AM, Reveley MA, Murray RM. Cerebral ventricular enlargement in non-genetic schizophrenia: a controlled twin study. Br J Psychiatry. 1984;144:89-93.

16. van den Heuvel MP, Fornito A. Brain networks in schizophrenia. Neuropsychol Rev. 2014;24(1):32-48.

17. Limongi $\mathrm{R}$, Jeon $\mathrm{P}$, Mackinley $\mathrm{M}$, et al. Glutamate and dysconnection in the salience network: neurochemical, effective connectivity, and computational evidence in schizophrenia. Biol Psychiatry. 2020;88(3):273-281.

18. Glausier JR, Lewis DA. Dendritic spine pathology in schizophrenia. Neuroscience. 2013;251:90-107.

19. Plitman E, Nakajima S, de la Fuente-Sandoval C, et al. Glutamate-mediated excitotoxicity in schizophrenia: a review. Eur Neuropsychopharmacol. 2014;24(10):1591-1605.

20. Fornito A, Yung AR, Wood SJ, et al. Anatomic abnormalities of the anterior cingulate cortex before psychosis onset: an MRI study of ultra-high-risk individuals. Biol Psychiatry. 2008;64(9):758-765.

21. Gallinat J, McMahon K, Kühn S, Schubert F, Schaefer M. Cross-sectional Study of glutamate in the anterior cingulate and hippocampus in schizophrenia. Schizophr Bull. 2016;42(2):425-433.

22. Reid MA, Salibi N, White DM, Gawne TJ, Denney TS, Lahti AC. 7T Proton magnetic resonance spectroscopy of the anterior cingulate cortex in first-episode schizophrenia. Schizophr Bull. 2019;45(1):180-189.

23. Poels EM, Kegeles LS, Kantrowitz JT, et al. Glutamatergic abnormalities in schizophrenia: a review of proton MRS findings. Schizophr Res. 2014;152(2-3):325-332.

24. Wang AM, Pradhan S, Coughlin JM, et al. Assessing brain metabolism with 7-T proton magnetic resonance spectroscopy in patients with first-episode psychosis. JAMA Psychiatry. 2019;76(3):314-323.

25. Choe BY, Suh TS, Shinn KS, Lee CW, Lee C, Paik IH. Observation of metabolic changes in chronic schizophrenia after neuroleptic treatment by in vivo hydrogen magnetic resonance spectroscopy. Invest Radiol. 1996;31(6):345-352.

26. Bustillo JR, Rowland LM, Mullins P, et al. 1H-MRS at 4 tesla in minimally treated early schizophrenia. Mol Psychiatry. 2010;15(6):629-636.

27. Merritt K, Perez-Iglesias R, Sendt KV, et al. Remission from antipsychotic treatment in first episode psychosis related to longitudinal changes in brain glutamate. npj Schizophr. 2019;5(1):12.

28. Henning A. Proton and multinuclear magnetic resonance spectroscopy in the human brain at ultra-high field strength: A review. Neuroimage. 2018;168:181-198. doi:10.1016/j. neuroimage.2017.07.017

29. Brandt AS, Unschuld PG, Pradhan S, et al. Age-related changes in anterior cingulate cortex glutamate in schizophrenia: A (1)H MRS Study at 7 Tesla. Schizophr Res. 2016;172(1-3):101-105.

30. Marsman A, Mandl RC, van den Heuvel MP, et al. Glutamate changes in healthy young adulthood. Eur Neuropsychopharmacol. 2013;23(11):1484-1490.

31. First MB, Williams JBW, Karg RS, Spitzer RL. Structured clinical interview for DSM-5 research version. Am Psychiatr Assoc Washingt DC. 2015.

32. Tkáč I, Gruetter R. Methodology of H NMR spectroscopy of the human brain at very high magnetic fields. Appl Magn Reson. 2005;29(1):139-157.

33. Near J, Edden R, Evans CJ, Paquin R, Harris A, Jezzard P. Frequency and phase drift correction of magnetic resonance spectroscopy data by spectral registration in the time domain. Magn Reson Med. 2015;73(1):44-50.

34. Bartha R, Drost DJ, Menon RS, Williamson PC. Spectroscopic lineshape correction by QUECC: combined QUALITY deconvolution and eddy current correction. Magn Reson Med. 2000;44(4):641-645.

35. van den Boogaart A, Ala-Korpela M, Jokisaari J, Griffiths JR. Time and frequency domain analysis of NMR 
data compared: an application to 1D 1H spectra of lipoproteins. Magn Reson Med. 1994;31(4):347-358.

36. Bartha R, Drost DJ, Williamson PC. Factors affecting the quantification of short echo in-vivo $1 \mathrm{H}$ MR spectra: prior knowledge, peak elimination, and filtering. NMR Biomed. 1999;12(4):205-216.

37. Wong D. MRI investigations of metabolic and structural brain changes in Alzheimer's disease and vitamin D deprivation. 2019.

38. Lin CH, Lin HS, Lin SC, Kuo CC, Wang FC, Huang YH. Early improvement in PANSS-30, PANSS-8, and PANSS-6 scores predicts ultimate response and remission during acute treatment of schizophrenia. Acta Psychiatr Scand. 2018;137(2):98-108.

39. Rybarczyk B. Social and Occupational Functioning Assessment Scale (SOFAS). Encyclopedia of Clinical Neuropsychology. 2018. doi:10.1007/978-3-319-57111-9_428

40. Singh SP, Cooper JE, Fisher HL, et al. Determining the chronology and components of psychosis onset: The Nottingham Onset Schedule (NOS). Schizophr Res. 2005;80(1):117-130.

41. Stentebjerg-Olesen M, Jeppesen P, Pagsberg AK, et al. Early nonresponse determined by the clinical global impressions scale predicts poorer outcomes in youth with schizophrenia spectrum disorders naturalistically treated with secondgeneration antipsychotics. J Child Adolesc Psychopharmacol. 2013;23(10):665-675.

42. Bush K, Kivlahan DR, McDonell MB, Fihn SD, Bradley KA. The AUDIT alcohol consumption questions (AUDIT-C): An effective brief screening test for problem drinking. Arch Intern Med. 1998;158(16):1789-1795. doi:10.1001/archinte.158.16.1789

43. Heatherton TF, Kozlowski LT, Frecker RC, Fagerstrom K -O. The Fagerström Test for Nicotine Dependence: a revision of the Fagerstrom Tolerance Questionnaire. $\mathrm{Br} J$ Addict. 1991;86(9):1119-1127. doi:10.1111/j.1360-0443.1991. tb01879.x

44. Skinner HA. The drug abuse screening test. Addict Behav. 1982;7(4):363-371.

45. IBM Corp. IBM SPSS Statistics for Windows, Version 26.0. 2019. 2019.

46. Kass RE, Raftery AE. Bayes factors. J Am Stat Assoc. 1995;90(430):773-795. doi:10.1080/01621459.1995.10476572

47. Keysers C, Gazzola V, Wagenmakers EJ. Using Bayes factor hypothesis testing in neuroscience to establish evidence of absence. Nat Neurosci. 2020;23(7):788-799. doi:10.1038/ s41593-020-0660-4

48. Morey RD, Rouder JN. BayesFactor: Computation of Bayes Factors for Common Designs. R Packag version 0912-42. 2018.

49. Sydnor VJ, Roalf DR. A meta-analysis of ultra-high field glutamate, glutamine, GABA and glutathione 1 HMRS in psychosis: implications for studies of psychosis risk. Schizophr Res. 2020;226:61-69.

50. Wenneberg C, Glenthøj BY, Hjorthøj C, et al. Cerebral glutamate and GABA levels in high-risk of psychosis states: a focused review and meta-analysis of $1 \mathrm{H}-\mathrm{MRS}$ studies. Schizophr Res. 2020;215:38-48.

51. Quiñones GM, MayeliA, Yushmanov VE, Hetherington HP, Ferrarelli F. Reduced GABA/glutamate in the thalamus of individuals at clinical high risk for psychosis. Neuropsychopharmacology. 2020;1-7. doi:10.1038/ s41386-020-00920-4

52. Shakory S, Watts JJ, Hafizi S, et al. Hippocampal glutamate metabolites and glial activation in clinical high risk and first episode psychosis. Neuropsychopharmacology. 2018;43(11):2249-2255.

53. Egerton A, Bhachu A, Merritt K, McQueen G, Szulc A, McGuire P. Effects of antipsychotic administration on brain glutamate in schizophrenia: a systematic review of longitudinal 1H-MRS studies. Front Psychiatry. 2017;8:66.

54. Birur B, Kraguljac NV, VerHoef L, et al. Neurometabolic correlates of 6 and 16 weeks of treatment with risperidone in medication-naive first-episode psychosis patients. Transl Psychiatry. 2020;10(1):15.

55. Cadena EJ, White DM, Kraguljac NV, et al. A longitudinal multimodal neuroimaging study to examine relationships between resting state glutamate and task related BOLD response in schizophrenia. Front Psychiatry. 2018;9:632.

56. Egerton A, Broberg BV, Van Haren N, et al. Response to initial antipsychotic treatment in first episode psychosis is related to anterior cingulate glutamate levels: a multicentre $1 \mathrm{H}-\mathrm{MRS}$ study (OPTiMiSE). Mol Psychiatry. 2018;23(11):2145-2155.

57. Kubota M, Moriguchi S, Takahata K, Nakajima S, Horita N. Treatment effects on neurometabolite levels in schizophrenia: a systematic review and meta-analysis of proton magnetic resonance spectroscopy studies. Schizophr Res. 2020;222:122-132.

58. Goto N, Yoshimura R, Kakeda S, et al. Six-month treatment with atypical antipsychotic drugs decreased frontal-lobe levels of glutamate plus glutamine in early-stage first-episode schizophrenia. Neuropsychiatr Dis Treat. 2012;8:119-122. 